\title{
ÉTUDE SUR LA SURVENANCE DES SINISTRES EN ASSURANCE AUTOMOBILE
}

\section{BRICHLER}

I. Distribution DES ASSURÉS SELON LE NOMBRE DES SINISTRES DANS UNE PÉRIODE DE TEMPS DÉTERMINÉE

On sait que la loi de Poisson simple représente mal la distribution des sinistres d'un groupe observé d'automobilistes du fait que tout groupe que l'on peut étudier en pratique, même s'il est composé d'assurés présentant des caractéristiques communes (même zone de circulation, même type de véhicule, même utilisation de ce véhicule, ...) est hétérogène quant aux autres caractéristiques et surtout quant au comportement personnel des assurés, élément dont l'influence sur les résultats du risque est prépondérante.

M. Delaporte a obtenu une représentation intéressante du phénomène en supposant que les sinistres d'un véhicule se répartissent suivant une loi de Poisson de moyenne donnée, et que les moyennes de chaque véhicule du groupe étudié se distribuent selon une loi de Pearson type III d'équation:

$$
d F(s)=\frac{a^{b}}{\Gamma(b)} e^{-a\left(s-s_{0}\right)}\left(s-s_{0}\right)^{b-1} d s
$$

où $\Gamma(b)$ est la fonction eulérienne de $2^{\text {ième }}$ espèce:

$$
\Gamma(b)=\int_{0}^{\infty} e^{-x} x^{b-1} d x
$$

$a, b$, et $s_{0}$ étant des paramètres dont la valeur est calculée en égalant les expressions des 3 premiers moments théoriques aux moments correspondants observés.

Cette formule conduit à des calculs assez longs.

M. Depoid a proposé une formule plus simple.

Si $n_{x}$ est, sur Io.00o véhicules, le nombre de ceux ayant eu dans l'année au moins $x$ sinistres, on a sensiblement:

$$
\log n_{x}=4-t_{x}
$$


$t$ étant donné par la relation

$$
\frac{\mathrm{I}}{t}=a+b F
$$

où $F=$ fréquence du groupe.

M. Depoid utilisait:

en province:

$$
\frac{I}{t}=0,68+3 F
$$

à Paris:

$$
\frac{\mathrm{I}}{t}=0,84+3,5 F
$$

M. Brichler a obtenu des résultats améliorés avec la formule suivante:

$$
t=\log \left(\mathrm{I}+\frac{\mathrm{I}}{F}\right)
$$

qui permet en outre des calculs très simplifiés. On en tire en effet, en appelant $N$ le nombre des véhicules du groupe et $N_{x}$ le nombre de ceux ayant eu exactement $x$ sinistres:

$$
\begin{aligned}
& N_{0}=\frac{N}{\mathrm{I}+F} \\
& N_{\mathbf{1}}=N_{0} \frac{F}{\mathrm{I}+F} \\
& N_{2}=N_{1} \frac{F}{\mathrm{I}+F} \\
& \ldots \\
& N_{x}=N_{x-1} \frac{F}{\mathrm{I}+F} \\
& \ldots
\end{aligned}
$$

On vérifie facilement que $\sum_{x=0}^{x-\infty} N_{x}=N$ 
et que: $\quad \sum_{x=0}^{x=\infty} x N_{x}=N F$

En sommant de $x$ à l'infini, on a le nombre de véhicules ayant $(x$ et +$)$ sinistres:

$$
N_{x} \text { et } t=N_{x-1} F
$$

L'expression ci-dessus est très commode en pratique, puisqu'elle permet de classer la totalité de la population, par exemple:

Assurés ayant o sinistre

Assurés ayant I sinistre

Assurés ayant 2 sinistres

Assurés ayant 3 sinistres et plus.

On démontre encore que le nombre moyen de sinistres des assurés ayant $(x$ et +$)$ sinistres est:

$$
x+F
$$

Par exemple, si la fréquence du groupe est $F=0,4$, la fréquence moyenne des assurés ayant ( 3 sinistres et + ) est 3,4 .

Ces formules simplifient considérablement les calculs liés aux sinistres (calculs de bonus-malus par exemple).

Applications numériques (exemples tirés de l'ouvrage de M. Depoid)

I. Sur un groupe de I.744 voitures de tourisme, usage promenade

\begin{tabular}{|c|c|c|c|c|c|}
\hline \multirow{4}{*}{$\begin{array}{l}\text { Nombre } \\
\text {-de } \\
\text { Sinistres }\end{array}$} & \multicolumn{5}{|c|}{ Nombre de véhicules } \\
\hline & \multirow{3}{*}{$\begin{array}{l}\text { Obser- } \\
\text { vations }\end{array}$} & \multicolumn{4}{|c|}{ Ajustements } \\
\hline & & $-\cdots-\cdots$ & $-\quad-\quad-$ & & $-\quad--$ \\
\hline & & $\begin{array}{c}\text { Poisson } \\
\text { simple }\end{array}$ & $\begin{array}{l}\text { Pearson III } \\
\text { (Delaporte) }\end{array}$ & $\begin{array}{c}\text { Formule } \\
\text { Depoid }\end{array}$ & $\begin{array}{l}\text { Formule } \\
\text { Brichler }\end{array}$ \\
\hline o & I.3I 6 & I. $26 \mathrm{I}$ & I. 316 & I. 3 IO & I.3 I 7 \\
\hline I & $3^{2} 3$ & 400 & 325 & $3^{22}$ & 322 \\
\hline 2 & $8 \mathrm{I}$ & 66 & 79 & 79 & 79 \\
\hline 3 & 18 & 7,2 & 19 & 19 & I9 \\
\hline 4 & 4 & 0,6 & 4,6 & 4,5 & 4,7 \\
\hline 5 & 2 & $\rho$ & 1,3 & 1,3 & $\mathrm{I}, 2$ \\
\hline 6 et + & - & $\mathcal{C}$ & 0,3 & 0,2 & 0,4 \\
\hline
\end{tabular}
en 1958. Fréquence moyenne: 0,324.

Les trois ajustements sont très bons. 
2. Exemples avec des fréquences extrêmes.

a) Renault $4 \mathrm{CV}$ - Province en I955 - groupe de 10.784 véhicules de fréquence moyenne 0,093

\begin{tabular}{c|r|r|r}
\multirow{2}{*}{ Néfr de sinistres } & \multicolumn{3}{|c}{ Nombre de véhicules } \\
\cline { 2 - 4 } & Observations & Poisson simple & Formule Brichler \\
\hline o & 9.835 & 9.799 & 9.839 \\
I & 843 & $9 \mathrm{I} \mathrm{I}$ & 836 \\
2 & $7 \mathrm{O}$ & 43 & $7 \mathrm{I}$ \\
3 & 6 & - & 7 \\
4 et + & - & & $\mathrm{I}$ \\
\hline
\end{tabular}

b) Citroen ID I9 - Paris en I958 - groupe de 2.224 véhicules de fréquence moyenne 0,679

\begin{tabular}{|c|c|c|c|}
\hline \multirow{2}{*}{ Néfr de sinistres } & \multicolumn{3}{|c|}{ Nombre de Véhicules } \\
\hline & Observations & Poisson simple & Formule Brichler \\
\hline o & I. 345 & I. $13^{\circ}$ & I. 325 \\
\hline I & 508 & 765 & 536 \\
\hline 2 & 228 & 260 & 217 \\
\hline 3 & 78 & $5^{8}$ & 83 \\
\hline 4 & 36 & Io & 36 \\
\hline 5 & I 7 & I & 14 \\
\hline 6 et + & I 2 & - & Io \\
\hline
\end{tabular}

La formule Brichler est un cas particulier de la formule Delaporte en faisant:

$$
a=\frac{\mathrm{I}}{F} \quad b=\mathbf{I} \quad s_{0}=\mathrm{o}
$$

\section{LIAISONS ENTRE LES FRÉQUENCES D'ANNÉES SUCCESSIVES}

M. Depoid a donné dans son ouvrage l'exemple suivant qui fait apparaître dans une population d'assurés, les liaisons entre les résultats des années successives:

r.250 contrats observés pendant 4 ans en ne conservant que les assurés restés dans la Société 18 mois au moins 


\begin{tabular}{c|c|c|c|c}
\hline \multirow{2}{*}{$\begin{array}{c}\text { Sinistres } \\
\text { la } \\
\text { Ière année }\end{array}$} & de 2ème année & de 3ème année & de 4ème année & de la 2e à la 4e \\
\hline 0 & 0,47 & 0,39 & 0,33 & $0,4 \mathrm{I}$ \\
$\mathrm{I}$ & 0,72 & 0,60 & 0,45 & 0,63 \\
2 et + & 1,06 & 0,90 & 0,58 & 0,94 \\
(moyenne 2,5) & & & & \\
\hline $\begin{array}{c}\text { Ensemble fré- } \\
\text { quence moyenne } \\
\text { o,67I }\end{array}$ & $0,63 \mathrm{I}$ & $0,5 \mathrm{I} 7$ & 0,386 & $0,55^{0}$ \\
\hline
\end{tabular}

(On observe une baisse de la fréquence générale du fait qu'on opère sur une population fermée).

M.Delaporte a proposé le modèle suivant:

Fréquence liée par le résultat $x$ d'une année:

$$
f / x=\frac{\int_{j 0}^{\infty} f P(x \mid f) d F(f)}{\int_{j 0}^{\infty} P(x / f) d F(f)}
$$

et si on a $x$ sinistres en $n$ années:

$$
f_{n / x}=\frac{\int_{f 0}^{\infty} f P\left(x_{1 / f}\right) \ldots P\left(x_{n / f}\right) d F(f)}{\int_{f 0}^{\infty} P\left(x_{1 / f}\right) \ldots P\left(x_{n / f}\right) d F(f)}
$$

$x=$ nombre annuel de sinistres

$$
P\left(x_{i / f}\right)=\frac{e^{-f} f^{x_{i}}}{x_{i} !}
$$

Sur l'exemple ci-dessus de M.Depoid, cette formulation permet de calculer les espérances mathématiques de zème, zème, 4ème années, liées par les fréquences de Ière année; en corrigeant de la tendance à la baisse signalée, on trouve:

\begin{tabular}{l|c|c|c}
\hline \multirow{2}{*}{$\begin{array}{c}\text { Sinistres } \\
\text { de la }\end{array}$} & \multicolumn{3}{|c}{ Fréquences liées } \\
\cline { 2 - 4 } & de 2ème année & de 3ème année & de 4ème année \\
\hline 0 & 0,469 & 0,384 & 0,287 \\
I & 0,710 & 0,582 & 0,434 \\
2 et + & I,066 & 0,874 & 0,652 \\
\hline
\end{tabular}


La concordance avec les résultats observés est satisfaisante, mais les calculs sont assez longs.

\section{Travaux récents du Groupement Technique Accidents}

Les observations récentes ont confirmé l'existence de liaisons entre les résultats d'années successives. Par exemple, sur I70.000 véhicules dont les résultats ont été observés en I960, I96I, I962 (en zone normale, tous usages), on a trouvé:

a)

\begin{tabular}{l|c|c|c|c|c}
\hline Sinistres la rère année: & 0 & I & 2 & 3 & 4 et + \\
\hline Fréquence de 2e année: & 0,17 & 0,37 & 0,59 & 0,85 & I, 15 \\
\hline
\end{tabular}

b)

\begin{tabular}{l|c|c|c|c|c|c|c|c}
\hline $\begin{array}{l}\text { Sinistres en z ans: } \\
\begin{array}{c}\text { Soit fréquence an- } \\
\text { nuelle: }\end{array}\end{array}$ & 0 & $\begin{array}{c}\mathrm{I} \\
0,5\end{array}$ & $\mathbf{I}$ & $\begin{array}{c}3 \\
1,5\end{array}$ & $\begin{array}{l}4 \\
2\end{array}$ & $\begin{array}{c}5 \\
2,5\end{array}$ & 3 & $\begin{array}{c}7 \mathrm{et}+ \\
3,5 \mathrm{et}+\end{array}$ \\
\hline $\begin{array}{l}\text { Fréquence de } 3 \mathrm{e} \text { an- } \\
\text { née: }\end{array}$ & $\mathrm{0, \textrm {I } 3 5}$ & 0,26 & $0,4 \mathrm{I}$ & 0,62 & $0,9 \mathrm{I}$ & $\mathrm{I}, 3 \mathrm{I}$ & $\mathrm{I}, 85$ & vers 3 \\
\hline
\end{tabular}

Le G.T.A. s'est proposé d'établir une formule simple rendant compte de ces liaisons.

I) Formule approchée:

En portant dans la formule des fréquences liées de M. Delaporte les valeurs des paramètres correspondant à la formule de distribution proposée par M. Brichler, elle se réduit à :

$$
f_{n} / x=F \frac{\mathrm{I}+x}{\mathrm{I}+n F} \quad \begin{aligned}
& F=\text { fréquence d'ensemble } \\
& x=\text { sinistres de } n \text { années }
\end{aligned}
$$

Appliquons cette formule aux résultats d'observations indiqués ci-dessus, pour lesquels $F=0,212$

\begin{tabular}{l|c|c|c|c|c}
\hline Sinistres la Ière année & 0 & I & 2 & 3 & 4 et + \\
\hline Fréquence de zème année & 0, I7 & 0,33 & 0,50 & 0,67 & 0,87 \\
\hline
\end{tabular}

La concordance n'est pas entièrement satisfaisante. 


\section{2) Formule développée:}

La formule indiquée plus haut a été améliorée récemment par MM. Acher et Thiry, au prix, bien entendu, d'une certaine complication.

Supposons une population fermée, dont la fréquence d'ensemble $F$ reste constante dans le temps (en fait, on sait qu'elle s'améliore on néglige ce facteur).

Pour un assuré de fréquence à l'origine supposée égale à $f_{0}{ }^{1}$ ) on va déterminer $f_{1}, f_{2}, \ldots$ fréquences des années $\mathbf{I}, 2, \ldots$ en fonction des $n$ années d'assurance écoulées et des sinistres observés pendant ces $n$ années. L'ajustement a été déterminé sur 3 années du fait qu'en Statistique Commune, on a des observations pour 3 années consécutives:

$$
60-6 \text { I-62 6I-62-63 63-64-65 }
$$

et de façon que, pour l'ensemble des assurés, la fréquence soit $F$ chaque année.

On pose, après $n$ années

$$
f_{n}+\mathrm{I}=f_{0} \frac{a+b s}{k+n}
$$

où $s=$ fonction de $x$ (nombre de sinistres pendant les $n$ années) et $a, b, k$ des paramètres dépendant de $f_{0}$.

On suppose que $\quad s=0$ si $x=0$.

Ajustement de a et de $k$ :

I) Pour $n=0$ qui entraîne $x=0$, on a la fréquence de rère année $f_{1}$ qu'on suppose $=f_{0}$

d'où

$$
a=k
$$

2) Pour $n=\mathrm{I}$, si on a eu o sinistre en rère année, on a $f_{2 / 0}=$ $=f_{0} \frac{a}{a+\mathrm{I}}$

( $f_{\mathrm{2} / \mathrm{0}}=$ fréquence 2 ème année si o sinistre la Ière année).

d'où

$$
a=\frac{\mathrm{I}}{\frac{f_{0}}{f_{2 / 0}}-\mathrm{I}}
$$

1) Fréquence d'origine $=$ fréquence supposée à la souscription du contrat. 
Pour différentes $f_{0}$ et en utilisant les 3 séries d'observation, on trouve un ajustement global assez bon en prenant:

$$
\log a=\frac{\mathrm{o}, \mathrm{I} 25}{f_{0}}
$$

Ajustement de b:

Supposons au départ que $s=x$, nombre de sinistres.

Vu la stabilité de $F$, si on a une population de $n$ assurés de fréquence de départ $f_{\mathbf{0}}$,

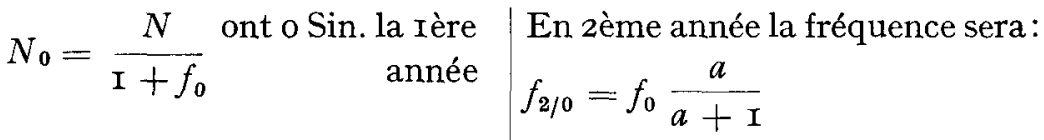

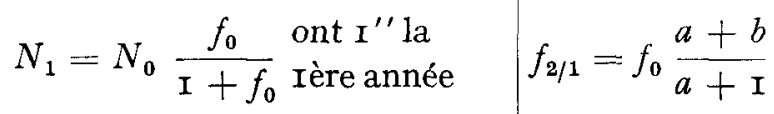

$$
\begin{aligned}
& \begin{array}{r}
N_{2} \text { et }+=N_{1} f_{0} \text { ont } 2 \text { Sin. et }+ \\
\text { la Ière année }
\end{array} f_{2 / 2+}=f_{0} \frac{a+b(2+\lambda)}{a+\mathrm{I}} \\
& \text { (Formules Brichler) } \\
& \text { où } \lambda=f_{0}
\end{aligned}
$$

En écrivant que $F$ se conserve:

$$
F=N f_{0}=N_{0} x f_{2 / 0}+N_{1} x f_{2 / 1}+N_{2 \text { et }}+x f_{2 / 2}+
$$

d'où l'on tire:

$$
b=\frac{\mathrm{I}}{f_{0}}
$$

En fait, l'expérience montre que les résultats se rapprochent davantage des observations en prenant

pour $n=\mathrm{I} \ldots \ldots \ldots, s=x$

pour $n=2 \ldots \ldots \ldots s=$ nombre de sin. Ière année $\times 0,8$

$+=$ nombre de sin. 2me année $\times \mathrm{I}, 2$

pour $n=3 \ldots \ldots \ldots s=(s$ ci-dessus pour $n=2) \times 0,8$

+ nombre de sin. 3me année $\times$ I,2

ce qui ne change pas le calcul ci-dessus (puisqu'on a le même numbre total de sinistres chaque année). Cette pondération correspond au fait observé que les sinistres plus récents pèsent plus lourd.

On peut encore améliorer en prenant:

$$
s=x \alpha^{x} \text { où } \alpha=\mathrm{I}+\varepsilon \varepsilon \text { étant de l'ordre de } 0, \mathrm{I}
$$


Il vient alors:

$$
b \simeq \frac{\mathbf{I}}{f_{0}(\mathbf{I}+\varepsilon)}
$$

En pratique, on a retenu au G.T.A. pour les calculs courants:

$$
f_{n+1}=f_{0} \frac{a+b s}{a+n} \quad s=x(\mathrm{I}, \mathrm{I})^{x}
$$

$$
\text { avec } \log a=\frac{0, \mathbf{I} 25}{f_{0}} \quad b=\frac{\mathrm{I}}{f_{\mathbf{0}(1,1)}}
$$

Exemple d'application pour $f_{0}=0,2 \mathrm{I} 2$

Il vient:

$$
f_{n+1}=f_{0} \frac{3,89+4,29 x(\mathrm{I}, \mathrm{I})^{x}}{3,89+n}
$$

On donne ici le rapport:

$$
\frac{f_{n+1}}{f_{0}} \times \text { roo }
$$

Dans chaque case la Ière ligne est la valeur observée en Statistique

\begin{tabular}{|c|c|c|c|c|c|}
\hline $\begin{array}{l}\text { Sinistres la } \\
2^{0} \text { année } \\
\text { Ière année }\end{array}$ & 0 & I & 2 & 3 & $4 \mathrm{et}+$ \\
\hline 0 & $\begin{array}{l}67 \\
66\end{array}$ & $\begin{array}{l}\text { I } 57 \\
161 \\
\end{array}$ & $\begin{array}{l}258 \\
277\end{array}$ & $\begin{array}{l}4 I 3 \\
4 I 6 \\
\end{array}$ & $\begin{array}{l}895 \\
773 \\
\end{array}$ \\
\hline I & $\begin{array}{l}125 \\
132 \\
\end{array}$ & $\begin{array}{l}231 \\
226\end{array}$ & $\begin{array}{l}350 \\
343\end{array}$ & $\begin{array}{l}570 \\
481\end{array}$ & $\begin{array}{l}795 \\
831\end{array}$ \\
\hline 2 & $\begin{array}{l}19 I \\
204\end{array}$ & $\begin{array}{l}300 \\
306\end{array}$ & $\begin{array}{l}442 \\
416\end{array}$ & $\begin{array}{l}595 \\
554\end{array}$ & $\begin{array}{l}966 \\
911\end{array}$ \\
\hline 3 & $\begin{array}{l}242 \\
299\end{array}$ & $\begin{array}{l}402 \\
394\end{array}$ & $\begin{array}{l}494 \\
510\end{array}$ & $\begin{array}{l}897 \\
649\end{array}$ & $\begin{array}{l}1212 \\
1006\end{array}$ \\
\hline $4 \mathrm{et}+$ & $\begin{array}{l}276 \\
532\end{array}$ & $\begin{array}{l}465 \\
634\end{array}$ & $\begin{array}{r}1080 \\
743\end{array}$ & $\begin{array}{l}915 \\
882\end{array}$ & $\begin{array}{l}2200 \\
1232\end{array}$ \\
\hline
\end{tabular}
Commune, la zème ligne est le résultat du calcul.

Indices de Fréquences de zeme année

\begin{tabular}{l|c|c|c|c|c}
\hline Sinistres la ı̀̀re année & o & I & 2 & 3 & 4 et + \\
\hline Fréquences de zème année & $\begin{array}{l}79 \\
80\end{array}$ & $\begin{array}{l}\text { I } 73 \\
\text { I } 76\end{array}$ & $\begin{array}{l}278 \\
292\end{array}$ & $\begin{array}{c}40 c \\
43^{\circ}\end{array}$ & $\begin{array}{l}722 \\
786\end{array}$ \\
\hline
\end{tabular}

Indices de fréquences de zeme annee

La concordance est bonne sauf pour la dernière case du second tableau qui concerne un très petit nombre d'assurés. 


\section{Annexe}

Les formules des pages 6 et 9 sont à rapprocher de celle de la „prime pondérée" proposée par M. Brichler dans une communication à l'Institut des Actuaires Français (Bulletin Juin I967 p. 20x). Il s'agit d'une prime variant chaque année en fonction du nombre de sinistres (comme la ,,prime modelée” de M. Delaporte).

Le principe en est le suivant:

La première année, l'assuré paye la prime $P_{0}$ correspondant au véhicule, à la zone, à l'usage, etc.... Après $n$ années, s'il $a$ eu $x$ sinistres, le coût moyen des sinistres étant $C$, la prime pourrait être

$$
\pi_{n}=\frac{x C}{n}
$$

En fait, nous estimons que la prime à percevoir réellement doit être un compromis entre-la prime de sa catégorie $(P o)$, prime moyenne établie au vu de statistiques provenant de nombreuses observations-et $\pi_{n}$, résultant de l'observation des sinistres individuels sur un nombre d'années en général petit et donc en partie aléatoire.

Le compromis peut être une moyenne pondérée:

$$
P_{n}=\frac{\alpha P_{0}+\beta \pi_{n}}{\alpha+\beta}
$$

La précision de $\pi_{n}$ s'améliorant chaque année, $\beta$ doit être une fonction croissante de $n$. Prenons $\beta=n$, d'où:

$$
P_{n}=\frac{{ }_{\alpha} P_{0}+x C}{\alpha+n}
$$

D'autre part, pour assurer un bon équilibre entre $P_{0}$ et $\pi_{n}$ il convient de prendre $\alpha$ d'autant plus grand que $P_{0}$ est petit.

En posant $\quad P_{0}=f_{0} x C, \quad$ prenons $X=\frac{k}{f_{0}}$

Il vient $\quad P_{n}=\frac{k+x}{k / f_{0}+n} C=\frac{k+x}{k+n f_{0}} P_{0}$

La formule de la page 6 correspond à $k=I$. 
Nous avons obtenu des résultats numériques très voisins de ceux de la prime modelée de M. Delaporte en prenant pour $k$ une valeur un peu plus grande que $\mathrm{I}(k=\mathrm{I}, 7)$, ce qui revient à donner un peu plus de poids à $P o$ qu'à $\pi_{n}$.

La formule de la page 9 correspond à $k=10 \frac{0,125}{f_{0}} x f_{0}$. 